\title{
An Overview of the Management of Mansonellosis
}

\author{
Thuy-Huong Ta-Tang (D) \\ Sergio LB Luz ${ }^{2}$ \\ James L Crainey ${ }^{2}$ \\ José M Rubio $\mathbb{D}^{3}$
}

'Malaria and NTDs Laboratory, National Centre of Tropical Medicine, Instituto de Salud Carlos III, Madrid, Spain;

${ }^{2}$ Laboratório de Ecologia de Doenças Transmissiveis na Amazônia, Instituto Leônidas e Maria Deane, Fundação Oswaldo Cruz, Manaus, Amazonas State, Brazil; ${ }^{3}$ Malaria \& Emerging Parasitic Diseases Laboratory, National Microbiology Center, Instituto de Salud Carlos III, Madrid, Spain
Correspondence: José M Rubio Malaria \& Emerging Parasitic Diseases Laboratory, National Microbiology Center, Instituto de Salud Carlos III, Cra. Majadahonda-Pozuelo Km 2,5, Majadahonda, Madrid, 28220, Spain

$\mathrm{Tel}+34918223420$

Fax +34915097034

Email jmrubio@isciii.es

James L Crainey

Laboratório de Ecologia de Doenças Transmissíveis na Amazônia, Instituto Leônidas e Maria Deane, Fundação

Oswaldo Cruz, 476 Rua Terezina,

Adrianópolis, Manaus, Amazonas State,

CEP 69057-070, Brazil

Email james.lee@fiocruz.br

\begin{abstract}
Mansonellosis is caused by three filarial parasite species from the genus Mansonella that commonly produce chronic human microfilaraemias: M. ozzardi, M. perstans and M. streptocerca. The disease is widespread in Africa, the Caribbean and South and Central America, and although it is typically asymptomatic it has been associated with mild pathologies including leg-chills, joint-pains, headaches, fevers, and corneal lesions. No robust mansonellosis disease burden estimates have yet been made and the impact the disease has on blood bank stocks and the monitoring of other filarial diseases is not thought to be of sufficient public health importance to justify dedicated disease management interventions. Mansonellosis's Ceratopogonidae and Simuliidae vectors are not targeted by other control programmes and because of their small size and out-door biting habits are unlikely to be affected by interventions targeting other disease vectors like mosquitoes. The ivermectin and mebendazole-based mass drug administration (iMDA and mMDA) treatment regimens deployed by the WHO's Elimination of Neglected Tropical Diseases (ESPEN) programme and its forerunners have, however, likely impacted significantly on the mansonellosis disease burden, principally by reducing the transmission of $M$. streptocerca in Africa. The increasingly popular plan of using iMDA to control malaria could also affect M. ozzardi parasite prevalence and transmission in Latin America in the future. However, a potentially far greater mansonellosis disease burden impact is likely to come from shortcourse curative anti-Wolbachia therapeutics, which are presently being developed for onchocerciasis and lymphatic filariasis treatment. Even if the WHO's ESPEN programme does not choose to deploy these drugs in MDA interventions, they have the potential to dramatically increase the financial and logistical feasibility of effective mansonellosis management. There is, thus, now a fresh and urgent need to better characterise the disease burden and ecoepidemiology of mansonellosis so that effective management programmes can be designed, advocated for and implemented.
\end{abstract}

Keywords: Mansonellosis, Mansonella perstans, Mansonella ozzardi, Mansonella streptocerca, Wolbachia, doxycycline

\section{Introduction}

Mansonellosis, and its synonym mansonelliasis, are medical terms used to describe a chronic filarial disease caused by parasites from the genus Mansonella. Mansonella genus parasites which cannot (or rarely) complete their life cycles using a human host, and therefore only cause zoonotic infections, are sometimes also considered to cause mansonellosis, but for the purposes review are not. ${ }^{1,2}$ For the purposes of this review, thus, mansonellosis infections are microfilaraemic, chronic, non-lethal infections caused by $M$. perstans, M. ozzardi or M. streptocerca. All three of these parasite species share very similar life-cycles and epidemiology (Figure 1) and are only known to cause mild, usually non- 


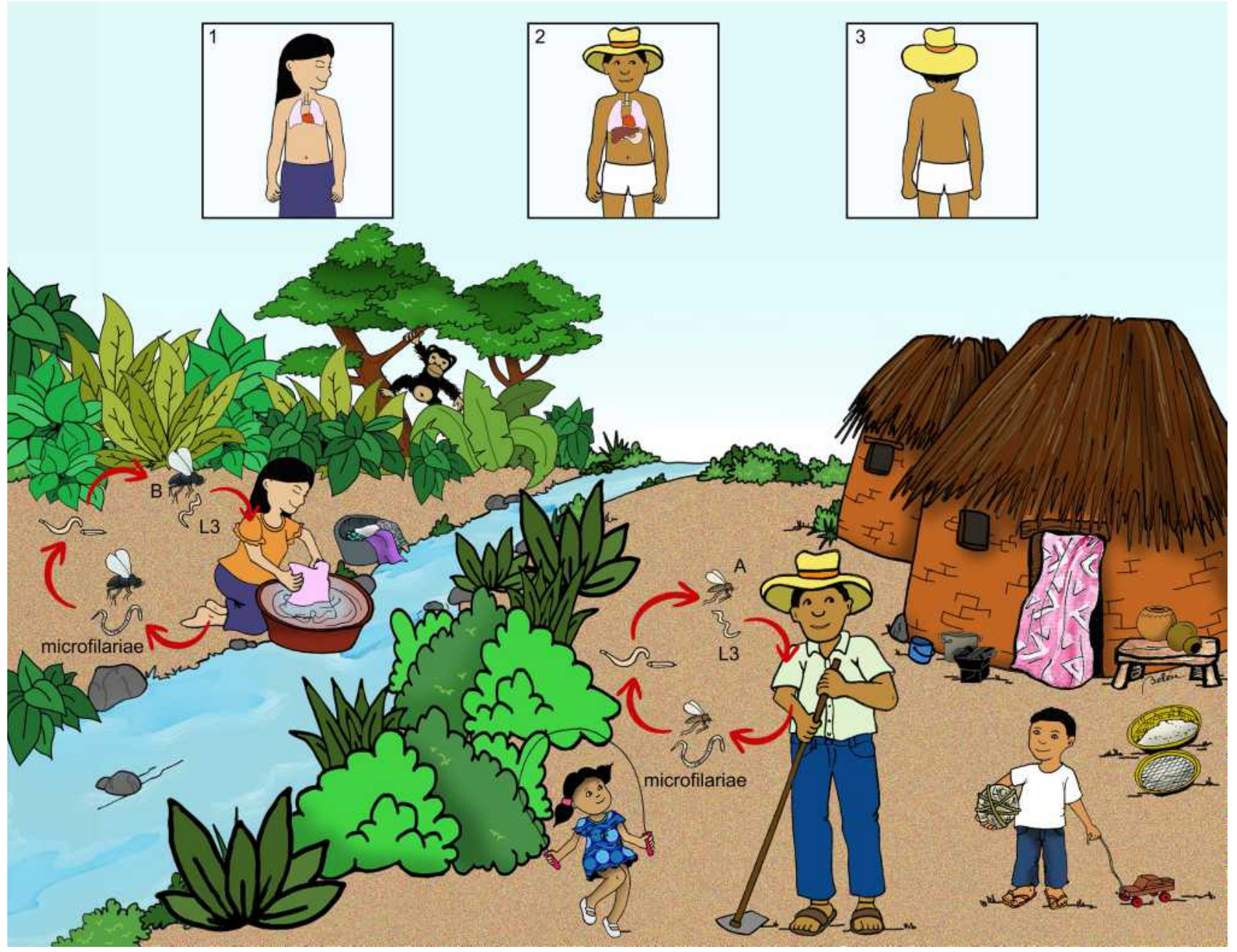

Figure I Integrated life cycle of the three Mansonella spp. An infected female blood-sucking arthropod genus Culicoides (A) for all the three Mansonella species, or Simulium (B) only for M. ozzardi] introduces third stage filarial larvae (L3) into the human host. The larvae develop into adult filariae, which commonly reside in the pleural cavity (I); the peritoneal cavity (2) or the subcutaneous dermal layer (3). The female worms produce microfilariae, which are found in peripheral blood (M. perstans and M. ozzardi) or found in the skin (M. ozzardi and M. streptocerca). An arthropod ingests the microfilariae during a blood meal. After ingestion, the microfilariae undergo two molts to become infective L3. The life cycle in which Culicoides vectors are involved can occur both in Latin American and African settings. Simuliid species are known to transmit M. ozzardi in Latin America.

specific symptoms, when they cause any symptoms at all. ${ }^{1,2}$ The disease affects tens, if not hundreds, of millions of the poorest people in Africa and Central and South America, but it is almost completely ignored by both national and international health agencies.

Although a number of mansonellosis symptoms including leg-chills, joint pains, headaches, fevers and corneal lesions have been robustly correlated with parasite infections, none have yet had their associated disease burden estimated. ${ }^{1,3}$ For example, while it is known that $M$. ozzardi infections cause corneal lesions in endemic areas of the Amazon region, it is not known if these lesions cause visual impairment or, if they do, to what extent those that have these lesions are visual impaired. ${ }^{4}$ This is, no doubt, in part related to the fact that other key disease burden measures have also not been estimated. Thus, while it is known other chronic filarial infections like onchocerciasis and loiasis can have a serious impact on an infected individual's life expectancy, no one has yet investigated whether mansonellosis infections impact on life expectancy. ${ }^{5,6}$ Without such estimates, it is difficult to calculate the disease burden caused by mansonellosis using standard measures. And as disease metrics are increasingly being used by research and public health programme funders to guide their financing decisions, a case can be made that mansonellosis research has become trapped in vicious circle of neglect. ${ }^{1,7}$ Research into the disease burden of mansonellosis has not been financed exactly because its disease burden is unknown. ${ }^{1}$ 
In contrast to mansonellosis research interest, which collapsed after a link between M. perstans and sleeping sickness was discarded at the beginning of $20^{\text {th }}$ century, onchocerciasis research interest has increased enormously over the last century. ${ }^{8,9}$ Substantial World Bank (WB) and World Health Organisation (WHO) financing for onchocerciasis research and control began in the early 1970s after epidemiologists established a strong link between the disease and visual impairment and blindness. ${ }^{8,9}$ This sustained research interest has led, indirectly, to the discovery of previously hidden pathologies, like its link to neurological disorders such as epilepsy, which only a minority of researchers previously recognised and which proved the onchocerciasis disease burden was underestimated well into the second decade of the $21^{\text {st }}$ century. ${ }^{10}$ This does not, of course, mean ipso facto that mansonellosis, also has hidden pathologies that would be uncovered if only the subject was more thoroughly investigated. It does, however, highlight that a chronic and really quite well-studied filarial disease, caused by a parasite with a very similar life-cycle to those which cause mansonellosis, can harbour a difficult to detect but not insignificant disease burden. Given how little research has been done on mansonellosis by comparison and that hitherto published mansonellosis epidemiological study designs would not have allowed any of the most important onchocerciasis disease burden estimates to be made (like the disease's impact on life-expectancy), there is, thus, scope to question if the disease's present perception as being largely benign is full justified or just a default position assumed in the absence of supporting data.

The public health importance of a disease is, of course, not necessarily limited to its defined disease burden. Until very recently there were no disease burden estimates for the filarial disease loiasis, which has a very similar epidemiological and clinical profile to mansonellosis. ${ }^{6,7}$ Unlike mansonellosis, however, it was included among the diseases listed in the WHO's Expanded Special Project for Elimination of Neglected Tropical Diseases (ESPEN) programme. ${ }^{11}$ This was largely because individuals heavily infected with loiasis (i.e. with high microfilaraemic loads) can suffer severe adverse events (SAE) when they are treated with ivermectin. ${ }^{12}$ Because of the broad distribution of loiasis in Africa, the WHO's ivermectin-based onchocerciasis and lymphatic filariasis mass drug administration (iMDA) programmes have been majorly impacted by the disease. ${ }^{10,12,13}$ While no associations between mansonellosis, mass drug administrations (MDAs) and SAEs have hitherto been reported, it has long been recognised that mansonellosis infections (like loiasis infections) can interfere with the epidemiological monitoring of onchocerciasis and lymphatic filariasis disease programmes. ${ }^{1,2,14-16}$ Similarly, the chronic nature of mansonellosis infections and the disease's broad global distribution, means the microfilariae from mansonellosis are a major contaminant of blood stocks in the developing world. ${ }^{17}$ Even though it appears that contaminated blood is transfused at lower rates than what would be expected from local prevalence levels, it appears that the microfilariae from mansonellosis infections are routinely transfused through-out the developing world. ${ }^{17,18}$ Although it is known that these transfusions cannot establish chronic infections, the microfilariae are known to be able to persist in the blood for over two years and could thus be contributing to transmission. ${ }^{18,19}$ At present, the WHO does not provide explicit guidance on how blood stocks contaminated with microfilariae should be treated. ${ }^{20}$ In a recent scoping expert panel review, it was concluded that it was unnecessary to screen for or mitigate against microfilariae contamination; however, not all experts share this view, and it appears some blood banks adopt the position that transfusion of blood contaminated in any way should be avoided. ${ }^{17,20-22}$

\section{Existing Helminth Disease Management Programmes}

Effective and co-ordinated helminth disease control programmes have been in operation for well over 70 years. ${ }^{13,23-27}$ Many of these programmes, which have in recent years focused on the use of anti-helminthic MDA and (to a lesser extent) vector control, have enjoyed substantial and sustained financing for almost a century. ${ }^{9,10,13,23}$ Typically, these programmes are co-ordinated by regional and national governments, often with financial and expert support from the WHO, the WB and various nongovernmental organisations (NGOs), like the Bill and Melinda Gates foundation. ${ }^{9,10,13,23}$ To date, most of this helminth disease control financing that has been directed towards filarial disease management has been spent on the management of lymphatic filariasis and onchocerciasis. ${ }^{9,10,13,23,24}$ Far less financing has been directed to the management of other filarial diseases, with most not directed to these two key filarial diseases being directed to dirofilarial disease control. ${ }^{28,29}$ Almost no dedicated 
financing has been directed to the management of mansonellosis. ${ }^{1,2}$

Unlike the financing that onchocerciasis and lymphatic disease control has benefited from, dedicated dirofilarial disease control has mostly come from the private sector through sponsorship of NGOs. ${ }^{28}$ NGOs like the American Heartworm Association; the European Society of Dirofilariosis and Angiostrongylosis, and the Companion Animal Parasites Council have worked toward curtailing the spread and transmission of dirofilarial disease through the promotion of slow-release prophylactic macrocyclic lactones treatment of companion animals. ${ }^{28}$ With support from their sponsors, which includes pharmaceutical companies involved with the fabrication and sale of the macrocyclic lactones these NGOs promote, these NGOs provide educational resources for veterinary professionals as well as dog and cat owners and also provide parasite distribution maps and animal treatment guidelines. ${ }^{30-32}$ Although the effectiveness of these prevention efforts has not been as carefully monitored as the WHO's onchocerciasis and lymphatic filariasis management programmes, the emergence and spread of Dirofilaria immitis ivermectin resistance in North America does suggest that the drugs being promoted are in sustained and widespread use in the regions that these NGOs target their use. ${ }^{33-35}$ It is, therefore, quite likely that these pet-owner driven interventions have had a significant impact on limiting dirofilarial disease transmission.

\section{The Impact of Vector Borne Disease Interventions on Mansonellosis}

It is also likely that dirofilarial disease transmission has been limited by vector control interventions targeting other vector-borne diseases with geographical distributions that overlap with dirofilarial disease. ${ }^{29}$ It is well recognised that in regions where lymphatic filariasis is transmitted by the same vector species being targeted for malaria control, these malaria control efforts can also reduce the regions lymphatic filariasis disease burden. ${ }^{24}$ As many of the most important mosquito vectors of dirofilarial disease (such as Culex pipiens quinquefasciatus, Aedes aegypti and Aedes albopictus) also transmit diseases that are targeted for control by other vector-borne disease programmes, ${ }^{29,36,37}$ it is likely that the transmission and public health impact of dirofilarial disease is being limited passively by the control of its vectors in regions where its distribution overlaps with lymphatic filariasis and arbovirus vector control programmes. ${ }^{29}$ And as such control programmes have been in widespread operation in areas where dirofilarial disease occurs for well over 70 years, ${ }^{37}$ it is quite likely that these programmes have had a non-trivial impact on dirofilarial disease also.

Mansonellosis, by way of contrast, is unlikely to have been significantly affected by vector control programmes targeting other diseases. In Africa and the Caribbean, mansonellosis is vectored by biting midges from the family Ceratopogonidae, which are not presently the targets of any other disease control programmes in these regions. ${ }^{1,2,36,38}$ Although $M$. ozzardi transmission in the Brazilian Amazon region involves many of the simuliid vectors involved in the region's onchocerciasis transmission, Amazon-region onchocerciasis control has not historically made use of vector control. ${ }^{39-41}$ This is in no small part because the region's large and inaccessible rivers make the river application of insecticides (larviciding), a far less practical approach to control onchocerciasis than it is in Africa and also because the outdoor biting and resting habits of its simuliid vectors render conventional adult-targeting interventions ineffective. ${ }^{36,39,40,42,43}$ Therefore, there are and never have been significant vector control programmes operating anywhere in the world that specifically target the vectors of mansonellosis. ${ }^{1,36,37,43}$

Although it is possible that the widespread use of insecticide residual spraying (IRS) for malaria, leishmaniasis or Chagas disease control, could be having a meaningful impact on the distribution of mansonellosis, this has not been investigated in any depth and is difficult to predict confidently owning to the existing paucity of data concerning the behavioural ecology of mansonellosis vectors. ${ }^{1,2,38,44}$ For example, in the Brazilian Amazon where IRS is an important tool for malaria control and mansonellosis is highly prevalent and caused by both $M$. perstans and $M$. ozzardi, not a single vector has hitherto been incriminated in $M$. perstans transmission. ${ }^{45-48}$ Thus while the outdoor biting habits of the simuliid filarial vectors of the Amazon are reasonably well characterised, ${ }^{40}$ the behavioural ecology of the South American ceratopogonids involved in M. ozzardi transmission (and presumably M. perstans transmission) is not well understood. ${ }^{1,2,40,45}$ Although, thus, one study recently showed that IRS treatment of homes and animal shelters can be used to decrease the abundance of adult Culicoides ${ }^{49}$ whether this approach will be effective for mansonellosis control will depend a great on the biting and resting habits of the most important vectors, which are still to be determined-not just in Latin America, but globally. ${ }^{1,2}$ 
The very small size of Culicoides and the outdoor biting habits of most simuliids also means that bed nets, which are used globally as an effective method of controlling mosquito-borne diseases like malaria and lymphatic filariasis, are unlikely to impact much on mansonellosis. ${ }^{37,50,51}$ Experiments using metal screen messing with aperture sizes much smaller than those typically used for mosquito bed nets showed that even very fine messing does not prevent the mansonellosis vector Culicoides furens from taking a blood meal. ${ }^{52}$ Experiments investigating whether Culicoides might be deterred by insecticide-treated messing have also proved disappointing and were congruent with what had already been established for phlebotomides sandflies. ${ }^{51}$ One large cluster control study investigating the impact of insecticide impregnated bed nets (with mesh apertures of sufficient size to allow phlebotomine vectors to pass), suggested that bed nets had little or no impact on the transmission of leishmaniasis. ${ }^{53}$ It is, thus, unlikely that the widespread use of bed nets in mansonellosis endemic regions is having any significant impact on mansonellosis transmission either in the new or old worlds.

\section{The Impact of MDA Programmes on Mansonellosis}

The extent to which the WHO's onchocerciasis and lymphatic filariasis MDA programmes have impacted on mansonellosis distribution is difficult to estimate. When the WHO launched its ESPEN programme in 2016, it treated around 131 million Africans with ivermectin as part of its onchocerciasis work. ${ }^{13}$ This new MDA programme followed on from sustained MDA treatment efforts which began in the 1980s and focused principally in West and Central Africa. ${ }^{9,10}$ As the ivermectin doses used in both the WHO's African lymphatic filariasis and onchocerciasis programmes are highly effective at clearing the microfilariae of M. streptocerca from the skin, ${ }^{54}$ it is likely these large-scale MDA programmes have had a significant impact on this parasite's transmission throughout the Central and West African regions where M. streptocerca occurs. ${ }^{1,2}$ In part because of the invasive nature of the skin snip assays used to evaluate the prevalence of M. streptocerca; however, the distribution of the M. streptocerca is very poorly mapped. ${ }^{1,2}$ With so little known about its vectors and their ecology, it is difficult to even predict their distribution as has been done for M. perstans. ${ }^{1,2,55}$ Molyneux et al $(2014)^{56}$ have argued that, because of the ecological effects of competitive exclusion, African human filarial parasites probably have quite limited overlapping distributions. Assuming this to be true, it seems likely that there are $M$. streptocerca endemic regions in West and Central Africa that are outside the ESPEN iMDA intervention areas that can act as M. streptocerca reservoirs to repopulate the intervention areas after ESPEN activities halt. Contemporary and historic onchocerciasis and lymphatic filariasis management programmes have used detailed epidemiological mapping to select treatment regimens and zones to minimise the risk of parasite recrudescence, in order to protect their intervention's hard won disease burden reductions. ${ }^{57-60}$ Despite this, however, there is no shortage of examples of lymphatic filariasis and onchocerciasis recrudescence events occurring following the removal of effective interventions. ${ }^{59,61,62}$ While, thus, there is evidence that M. streptocerca transmission is and has been significantly impacted by iMDA programmes (see, for example, Ta et al $2018)^{63}$ and it is likely that the parasite may have been eliminated from some MDA treatment zones, it is also very likely that, because its epidemiological dynamics are similar to other filarial diseases, the parasite will repopulate most of these zones after MDA has been halted., ${ }^{2,27}$ Any passive mansonellosis disease burden gains from African iMDA programmes are likely to be only temporary, unless targeted action is taken to preserve them.

Although the WHO's standard recommend treatment for lymphatic filariasis (Diethylcarbamazine [DEC] with albendazole) would be expected to clear M. perstans microfilariae from those treated with it, this treatment regime is almost never used anywhere that $M$. perstans is prevalent. ${ }^{1,64}$ Because of the risks associated in using DEC on someone infected with $O$. volvulus, the WHO almost exclusively uses ivermectin with albendazole (which does not efficiently clear $M$. perstans parasitaemias) for its lymphatic filariasis MDA programmes throughout Africa. ${ }^{1,64}$ And as lymphatic filariasis has only a very limited distribution in the areas of the Amazonian rainforest where M. perstans occurs in Latin America, it is unlikely that its transmission is being affected by lymphatic filariasis control measures in any meaningful way in the New World either. ${ }^{1,45,48}$ Latin American onchocerciasis iMDA programmes would similarly not be expected to have any impact on $M$. perstans transmission and to have only a limited impact on $M$. ozzardi as it is presently only being deployed in the Yanomami territory where approximately 30,000 people are thought to be at risk of 
onchocerciasis infections and far fewer are treated. ${ }^{27,65}$ And although the ESPEN's doxycycline treatment regimens would be expected to cure those treated with M. perstans, as this tool is usually being deployed in test and treat way (and M. perstans infected individuals are not being treated unless they are also infected with onchocerciasis or lymphatic filariasis), in areas where loiasis and onchocerciasis co-occur this approach is also probably having only an extremely limited impact on African mansonellosis. ${ }^{66}$

It is estimated that the global population is now taking more than 1 billion drug treatments for Soil Transmitted Helminths (STH) every year. ${ }^{13}$ Most of these treatments are delivered through MDA programmes targeting school-aged children and use either albendazole, mebendazole or ivermectin. ${ }^{13}$ As STH occur almost everywhere that mansonellosis does and the dosage of mebendazole used to treat them (when it is used) would be expected to have an impact on $M$. perstans parasitaemias, it can be reasonably concluded that $M$. perstans transmission is likely to have been suppressed in areas where STH mMDA programmes have operated. ${ }^{1,13,67,68}$ It is, however, important to note that although STH MDA programmes have been carried out for almost a century, they typically only include a small proportion of the population (school age children) and typically do not target the same areas for sustained periods. ${ }^{1,2,13,67,68}$ Extrapolating loosely from onchocerciasis modelling, which suggest high-level treatment coverage is key to transmission suppression, while one would expect STH mMDA to suppress $M$. perstans transmission it would not be expected to break it anywhere that it was used. ${ }^{69}$ Although, thus, it has been estimated that more than 100 million people are infected with $M$. perstans in Africa alone and mMDA has been used widely to control STH in these areas for decades, it is unlikely these programmes have had a greater impact on the disease burden of mansonellosis than the onchocerciasis and lymphatic filariasis iMDA programmes, which clear M. streptocerca microfilariae for sustained periods and thus prevent transmission and new infections. ${ }^{13,38}$ Consistent with this, comparisons between historic and contemporary M. perstans blood smear surveys from Africa suggest that $M$. perstans prevalence has been stable since records started getting taken. ${ }^{1,13,38}$ It is, however, possible that these programmes have promoted M. perstans anthelmintic resistance.
Even though MDA programmes are not presently being used for malaria control the idea of using iMDA to control malaria has steadily been gaining support since it was first proposed in the 1980s (The ivermectin roadmappers 2020). ${ }^{70}$ While most proposals are operationally very similar to the onchocerciasis and lymphatic filariasis iMDA programmes (but without the 20 year annual treatment commitment), the way they work, by killing or reducing the life span of mosquitoes feeding on ivermectin laced blood, is fundamentally different. ${ }^{70,71}$ As most of the ivermectin doses and treatment regimens that are being tested in clinical trials are similar to those used in the ESPEN iMDA programmes in West and Central Africa, it is likely that if iMDA malaria programmes are rolled-out in these regions, these programmes will also have a significant and synergistic impact on $M$. streptocerca in these regions. ${ }^{70}$ And as $M$. ozzardi microfilariae are also highly susceptible to ivermectin, ${ }^{72}$ it is likely that if sustained iMDA is used in the Amazon for malaria control (as is being proposed Perez-Garcia et al 2018, Yudi et al 2018), ${ }^{71,73}$ M. ozzardi transmission will be impacted in a similar way to which $M$. streptocerca transmission is presently being affected in Africa. However, whether malaria iMDA does get rolled-out on a large scale in either of these regions seems likely to depend a great deal on whether such an approach can gain ethical approval and community support. Recent experiments suggest that the ivermectin doses currently being proposed for these programmes do not have a positive clinical benefit to individuals infected with malaria, complicating ethical advocacy for the programme. ${ }^{70,74}$ There is also little to no clinical benefit to the individuals in onchocerciasis and lymphatic filariasis endemic areas that participate in iMDA programmes. These programmes, which also require extremely high levels of community participation to function, have, however, been operating successfully throughout the globe for decades. ${ }^{9,10,24,69}$ Given the success of the onchocerciasis and lymphatic filariasis control programmes and the fact that iMDA malaria control trials are also underway, it appears that ethical and community participation concerns may not prove to be a barrier to the roll-out of this sort of programme. On the other hand, iMDA non-compliance does appear to be on the rise at least in Africa; ${ }^{66}$ should this rise continue, all future iMDA programmes, including new malariatargeting programmes, could be jeopardised. 


\section{Existing Use of Anti-Wolbachia Treatments in Filarial Disease Management}

Presently, the WHO's use of doxycycline is primarily focused in regions of Africa where onchocerciasis and loiasis are co-endemic and thus in regions where ivermectin cannot always be used safely because of the risk of SAEs associated with the ivermectin treatment of individuals with high Loa Loa microfilaraemic loads. ${ }^{9,66}$ Part of the reason why the WHO is reluctant to deploy doxycycline more widely in its control programmes is the prior cost-effective successes at reducing transmission and disease morbidity using iMDA as a principle or sole intervention. ${ }^{9,10,27}$ These past successes support the hypothesis that the elimination of these diseases can be achieved with iMDA and only a limited amount of alternative interventions and thus that filarial disease management resources should be focused on attaining these goals using existing tools rather than developing potential alternatives. ${ }^{9,10,24,64}$ And although it is widely accepted that additional tools will be needed to complement iMDA for the WHO to achieve its ambitious filarial disease elimination targets, insect vector targeting tools (such as insecticideimpregnated bed nets for lymphatic filariasis control and organophosphate ground larviciding [treating of specific rivers with temephos] for the control of onchocerciasis) are likely to be more often deployed as complementary interventions owing to their proven utility in filarial disease control. ${ }^{9,10,24}$ In addition to this, the fact that doxycycline regimes used for filarial disease treatment also prevent the inclusion of pregnant women and young children under 8 (who can be affected by neurological disorders) from being included in treatment programmes, means that while doxycycline does have a good safety profile, when used alone, it may not always be possible to achieve the treatment coverage levels required to break filarial parasite transmission. ${ }^{9,10,24}$ Unfortunately, this is compounded by the fact that microfilariae persist in a doxycycline-treated individual's blood for several months after they are treated and even though experimental work suggests that Wolbachia-free microfilariae cannot develop properly in their insect vector hosts, it is unlikely that doxycycline treatment will be as effective as existing treatments at breaking filarial parasite transmission and thus that any doxycycline treatment should be complemented with traditional anti-filarial therapeutics (ivermectin for onchocerciasis, DEC or albendazole [or both] for lymphatic filariasis) wherever possible. ${ }^{9,10,24}$
Perhaps, however, the factor most limiting a greater use of doxycycline treatment regimes in filarial disease management is that effective filarial disease treatment with doxycycline requires daily or twice daily administration of the drug for periods of between 4 to 6 weeks. These longterm regimens make doxycycline-based MDA (dMDA) programmes much more logistically difficult and expensive to implement than iMDA programmes. Test-and-treat strategies can reduce the expense of dMDA (by preventing public health professionals investing considerable time treating individuals that are not infected with the targeted filarial parasite), but even when such approaches are adopted dMDA is still far more expensive and complex to implement than iMDA. ${ }^{9,10,24,66}$ And even though costeffective diagnostic tools have been developed to assist with test-and-treat dMDA approaches, existing tools are not all field-friendly and not always accessible everywhere MDA is needed. ${ }^{1}$

Although thus iMDA strategies offer little clinical benefit to any individual accepting treatment and none to those uninfected (they offer a benefit to the whole treated community through reducing the number of new infections in any treated area), they are unlikely to be replaced by ethically more sound doxycycline-based test and treat strategies that offer a potential cure to those treated as well as, in the case of lymphatic filariasis, sometimes offering a direct clinical benefit by providing relief from the symptoms caused by their infections. ${ }^{9,10,24,66}$ New Wolbachia-targeting drugs with short-course treatment regimens could however change this scenario by making curative (anti-Wolbachia) treatment regimens cheaper and more practical to deliver than doxycycline-based MDA regimes. ${ }^{9,75-77}$ In recognition of the potential of Wolbachia targeting drugs for accelerating the elimination of filarial diseases a team at the Liverpool School of Tropical Medicine, set up the anti-Wolbachia (A-WOL) consortium with the objective to develop Wolbachia-targeting therapeutics with good efficacy and safety profiles and with the potential to replace the present anti-helminthic treatment drugs being used in MDA, which break parasite transmission but do not offer clear clinical benefits to those that are treated with them., ${ }^{9,75-78}$

\section{Novel Anti-Wolbachia Drug Discovery, Engineering and Testing}

Using insect cell lines and robotics, the A-WOL consortium and its partners have tested more than 2 million pharmaceutically active compounds from pharmaceutical 
companies' chemical compound libraries for their activity against Wolbachia endosymbionts and their toxicity to their eukaryotic cellular hosts. ${ }^{9,35,75,79}$ From these initial screens around 22,500 chemical compounds with an ability to kill Wolbachia, but not their host cells, were identified. ${ }^{35}$ These compounds were then subjected to a cheminformatic screen to identify potential compounds that should be prioritised for animal testing. ${ }^{35}$ This screen is considered the toxicology profiles of the compounds as well as their metabolic stability, physiochemical properties and the molecular weight. ${ }^{35}$ Before being tested on animal models, some of these selected compounds were also tested for activity against Brugia malayi microfilariae and adults using in vitro assays. ${ }^{35}$ In vivo testing on animal models identified a number of chemical compounds which can kill adult filarial parasites in less than seven days of treatment. ${ }^{35}$ One particularly interesting compound that was identified by A-WOL compound screening was Tylosin A, which was already licenced for medical use on animals. ${ }^{9,75,77,79}$ In experiments using this drug against filarial parasites in animal model systems, however, it was noted that injections were far more effective than oral treatments and it was proposed that the drug's high solubility hampered its absorption from the gut lumen making it unsuitable for oral delivery. ${ }^{35,75,77}$ To improve this drug 's oral bioavailability around 150 Tylosin A analogous, with carbamates or bulky esters, were designed, synthesised and tested on animal models. ${ }^{35,75,77,79}$ Testing these analogues identified chemicals with both improved oral bioavailability and indeed potency against Wolbachia. A seven-day treatment regime with one of these analogues, ABBV-4083, was shown to be effective against both lymphatic filariasis and onchocerciasis animal models and was thus selected for human clinical trials. ${ }^{9,35,75,77,79}$ Having successfully completed Phase I clinical trials, this drug is presently being tested for its utility against onchocerciasis infections in Phase II clinical trials. ${ }^{9,35,75,77,79,80}$ A-WOL's compound screening and that of their Californian partners testing is, however, continuing with very promising results..$^{9,35,75,77,79}$ Most impressively of all, single doses of the quinazolines CBR417 and CBR490 on the Litomosoides sigmodontis rodent model were recently shown to eliminate $>99 \%$ the parasites Wolbachia $^{81,82}$ While these drugs still need to move on to clinical trials, these single-dose anti-Wolbachia curative therapies clearly have the potential to replace existing drugs used in almost all MDA programmes presently in operation for onchocerciasis and lymphatic filariasis management. ${ }^{9,35,75,78}$

\section{The Prospect of Using Anti-Wolbachia Therapeutics for Mansonellosis Treatment}

While anti-Wolbachia treatments have been proven to be effective for almost all the human and animal filarial parasites they have been tested against, it was not immediately obvious that they would be effective for mansonellosis. Early reports suggested that at least some M. perstans parasites did not harbour Wolbachia, which strongly suggested Mansonella genus parasites did not need their Wolbachia to complete their life cycles and thus that they would not make good drug targets. ${ }^{83}$ This conclusion was also supported further by phylogenetic analysis, which has consistently grouped Mansonella Wolbachia in the only supergroup that contains both arthropod and filarial parasite infecting strains. ${ }^{48,84,85}$ As the vast majority of the arthropod-infecting Wolbachia strains are reproductive parasites that can be removed from their hosts without significant fitness costs, the presence of arthropod Wolbachia in superclade F would typically be seen as suggesting that members of Wolbachia supergroup are dispensable for their hosts and thus poor drug targets for filarial treatments. ${ }^{84}$

The evidence that Wolbachia could be effectively targeted for mansonellosis treatment, has, however, been growing steadily. Most importantly, clinical trials have repeatedly shown doxycycline is an effective treatment for M. perstans infections. ${ }^{86,87}$ One of these studies and detailed studies investigating the prevalence of Wolbachia in $M$. perstans clinical samples led to the hypothesis that the initial perceived absence of Wolbachia from certain M. perstans samples could be explained by limitations in the sensitivity of the PCR assays used to detect them. ${ }^{88}$ Consistent with this theory and that idea that $M$. ozzardi also has a mutualistic relationship with its host (and could therefore be effectively targeted with anti-Wolbachia therapeutics), a recent survey of $48 \mathrm{M}$. ozzardi positive blood samples found that Wolbachia could be detected in all the samples if enough different Wolbachia detecting PCR assays were deployed. ${ }^{89}$ Although the occurrence of Wolbachia in M. streptocerca still needs to be established before it can be proposed that they too can be treated with anti-Wolbachia therapeutics, Wolbachia has now been found in multiple species from the genus suggesting these infections were established before the radiation of the genus and thus that they will likely be found in M. streptocerca too. ${ }^{90}$ Importantly and surprisingly too, it 
appears increasingly apparent that the arthropod-infecting supergroup F Wolbachia strain $w$ Cle is a mutualist which provides its arthropod host with significant fitness benefits. $^{91,92}$ Most existing evidence thus now suggests that Wolbachia supergroup F strains can be targeted for effective mansonellosis treatment and management.

The prospect of using anti-Wolbachia treatments for mansonellosis is particularly important in the Northern Amazon region, where Mansonella perstans appears to commonly and possibility exclusively occur in coinfections with $M$. ozzardi. ${ }^{48}$ As ivermectin treatment is ineffective at clearing $M$. perstans and mebendazole treatment is ineffective at clearing $M$. ozzardi, there is no tried and tested MDA treatment regime effective for preventing mansonellosis transmission in these areas. ${ }^{1,48}$ Although a combination treatment of ivermectin and mebendazole could be expected to be effective for this disease, a shortcourse curative Wolbachia-targeting treatment could be seen as far more preferable given both treatment regimens would need to be trailed before being rolled-out.

\section{Future Prospects for Mansonellosis Disease Management}

Despite being one of the world's most prevalent chronic infectious diseases, mansonellosis is not specifically being targeted for control by the WHO, WB or any NGO or any major national government control programmes. ${ }^{1,2}$ Even though dirofilariasis is less prevalent and not known to have worse pathologies in humans than mansonellosis, far more is done to control it. Whether the dirofilarial intervention strategies or financial models for the disease's management could or should be used for mansonellosis management is, however, debatable especially given the differences in the two diseases' global distribution. Clear public health benefits have been obtained in the same poverty-stricken regions where mansonellosis occurs following successful public health advocacy for STH. Given that the pathologies and disease burden of STHs are more similar in their severity to those caused by mansonellosis than those of onchocerciasis and lymphatic filariasis, an argument could be made that mansonellosis management could benefit from following the STH health advocacy strategies more closely. However, as neglected tropical diseases are increasingly being grouped together and controlled in synergistically designed strategies, it is arguably in this context that future mansonellosis control strategies should be envisaged.
The WHO is presently planning the elimination of onchocerciasis, lymphatic filariasis, loiasis and STHs within the context of its ESPEN programme. As discussed above, iMDA and mMDA are a major feature of these plans and are likely to impact on the distribution of $M$. perstans and $M$. streptocerca in Africa. It is thus disappointing that this programme has not included mansonellosis within its remit so that the programme's interventions are designed to maximise mansonellosis disease burden reductions in Africa. Although the ESPEN programme is unlikely to have much impact on Latin American mansonellosis, plans to control malaria with iMDA could have. Such a project could be expected to have a major impact on mansonellosis in the Brazilian Amazon where it is co-endemic with malaria and where M. ozzardi is the predominant etiological agent. However, without a clearly defined disease burden, it is unlikely that iMDA malaria control project designs will try to maximise or even consider the potential impact they will have on mansonellosis.

To obtain a more clearly defined disease burden, epidemiological studies are going to need to be more in depth and better designed so that the results obtained can be used in standardised disease burden calculations. The link between onchocerciasis and blindness was shown by associating high microfilaraemic loads with blindness - if all infections are considered the association is more difficult to detect. Epidemiological studies investigating mansonellosis have, however, almost never investigated a link between microfilaraemic load or exposure to infectious vector bites with disease pathology and often miss the lightest infections because they do not use PCR diagnosis. ${ }^{1,2,93}$ Recently developed real-time PCR assays have the potential to address these issues, but have not yet been used enough to investigate the diseases pathology. ${ }^{48,63}$ Similarly, although novel methods for mansonellosis vector trapping, which could be used to investigate a link between infectious bites and disease pathology, have been developed, they have still not yet been deployed for any type of mansonellosis epidemiological study. ${ }^{94}$ Similarly, although techniques for the isolation and genotyping of individual filarial individual microfilariae, which could improve our understanding of mansonellosis parasite population dynamics enormously, have developed, they have not yet been used in epidemiological studies of mansonellosis. $^{95}$

Of course, as discussed above, the public health importance of mansonellosis is not limited to its directly attributed disease burden. ${ }^{1,2,15}$ And while the impact chronic 
mansonellosis infections have on vaccination programmes has not yet been investigated in any depth there is growing evidence from other filarial disease studies to suggest it could be non-trivial. ${ }^{96,97}$ Thus, while mansonellosis is evidently not seen to be of sufficient public health importance to justify the financial support required for a 20-year-long iMDA-based programme like those used by the ESPEN programme, this does not mean that its management could not be justified by more cost-effective methods. With the advent of Wolbachia-targeting single-dose curative filarial treatments looking increasing close, the logistical and finical viability of mansonellosis management is changing dramatically and for the better. Thus, beyond the need for more studies to investigate the disease burden of mansonellosis, there is an urgent need to improve understanding of the epidemiology of the disease so that effective antiWolbachia therapeutic-based MDA programmes can be designed and deployed should such drugs become available for mansonellosis management.

\section{Abbreviations}

A-WOL, anti-Wolbachia; DEC, diethylcarbamazine; ESPEN, Expanded Special Project for Elimination of Neglected Tropical Diseases; IRS, insecticide residual spraying; iMDA, ivermectin-based MDA; MDA, mass drug administration; mMDA, mebendazole-based MDA; NGOs, non-governmental organisations; PCR, Polymerase chain reaction; SAE, severe adverse events; STH, Soil-Transmitted Helminths; WB, World Bank; WHO, World Health Organization.

\section{Acknowledgments}

We would like to express our special thanks to María Belén García Fernández for helping us to draw the lifecycle of Mansonella species. JLC and SLBL also gratefully acknowledge support from the Fundação de Amparo à Pesquisa do Estado do Amazonas (FAPEAM; 062.01282/2018 and 002.00200/2019). And JLC would like to acknowledge support he receives from a Conselho Nacional de Desenvolvimento Científica e Tecnológico (CNPq) productivity grant. THTT is funded by a Sara Borrell contract from the Instituto de Salud Carlos III.

\section{Disclosure}

The authors report no conflicts of interest in this work.

\section{References}

1. Ta-Tang T-H, Crainey JL, Post RJ, Luz SL, Rubio JM. Mansonellosis: current perspectives. Res Rep Trop Med. 2018;9:9-24. doi:10.2147/RRTM.S125750

2. Medeiros JF, Crainey JL, Pessoa FAC, Luz SLB. Mansonelliasis. In: Marcondes CB editor. Arthropod Borne Diseases. Springer International Publishing; 2017:405-426. doi:10.1007/978-3-31913884-8_26.

3. Ferreira MU, Crainey JL, Luz SLB. Mansonella ozzardi. Trends Parasitol. 2021;37(1):90-91. doi:10.1016/j.pt.2020.03.005

4. Vianna LMM, Martins M, Cohen MJ, Cohen JM, Belfort R. Mansonella ozzardi corneal lesions in the Amazon: a cross-sectional study. BMJ Open. 2012;2:6. doi:10.1136/bmjopen2012-001266

5. Little MP, Breitling LP, Basáñez M-G, Alley ES, Boatin BA. Association between microfilarial load and excess mortality in onchocerciasis: an epidemiological study. Lancet. 2004;363 (9420):1514-1521. doi:10.1016/S0140-6736(04)16151-5

6. Chesnais CB, Nana-Djeunga HC, Njamnshi AK, et al. The temporal relationship between onchocerciasis and epilepsy: a population-based cohort study. Lancet Infect Dis. 2018;18(11):1278-1286. doi:10.1016/S1473-3099(18)30425-0

7. Veletzky L, Hergeth J, Stelzl DR, et al. Burden of disease in Gabon caused by loiasis: a cross-sectional survey. Lancet Infect Dis. 2020;20 (11):1339-1346. doi:10.1016/S1473-3099(20)30256-5

8. Crump A, Morel CM, Omura S. The onchocerciasis chronicle: from the beginning to the end? Trends Parasitol. 2012;28(7):280-288. doi:10.1016/j.pt.2012.04.005

9. Brattig NW, Cheke RA, Garms R. Onchocerciasis (River Blindness) more than a century of research and control. Acta Trop. 2020;105677. doi:10.1016/j.actatropica.2020.105677

10. Colebunders R, Basáñez M-G, Siling K, et al. From river blindness control to elimination: bridge over troubled water. Infect Dis Poverty. 2018;7(1):21. doi:10.1186/s40249-018-0406-7

11. ESPEN. ESPEN | expanded Special Project for Elimination Neglected Tropical Diseases. Available from: https://espen.afro.who. int/. Accessed March 29, 2021.

12. Boussinesq M, Gardon J, Gardon-Wendel N, Chippaux J-P. Clinical picture, epidemiology and outcome of Loa-associated serious adverse events related to mass ivermectin treatment of onchocerciasis in Cameroon. Filaria J. 2003;2(1):S4. doi:10.1186/1475-2883-2-S1-S4

13. Bundy DAP, Appleby LJ, Bradley M, et al. 100 years of mass deworming programmes: a policy perspective from the world bank's disease control priorities analyses. $A d v$ Parasitol. 2018;100:127-154. doi:10.1016/bs.apar.2018.03.005

14. Fischer P, Bamuhiiga J, Büttner DW. Occurrence and diagnosis of Mansonella streptocerca in Uganda. Acta Trop. 1997;63(1):43-55. doi:10.1016/s0001-706x(96)00607-9

15. Tang T-HT, López-Vélez R, Lanza M, Shelley AJ, Rubio JM, Luz SLB. Nested PCR to detect and distinguish the sympatric filarial species Onchocerca volvulus, Mansonella ozzardi and Mansonella perstans in the Amazon Region. Mem Inst Oswaldo Cruz. 2010;105 (6):823-828. doi:10.1590/s0074-02762010000600016

16. Alhassan A, Li Z, Poole CB, Carlow CKS. Expanding the MDx toolbox for filarial diagnosis and surveillance. Trends Parasitol. 2015;31(8):391-400. doi:10.1016/j.pt.2015.04.006

17. Abrahim CMM, Py-Daniel V, Luz SLB, Fraiji NA, Stefani MMA. Detection of Mansonella ozzardi among blood donors from highly endemic interior cities of Amazonas state, northern Brazil. Transfusion. 2019;59(3):1044-1051. doi:10.1111/trf.15101

18. Nutman TB. Experimental infection of humans with filariae. Rev Infect Dis. 1991;13(5):1018-1022. doi:10.1093/clinids/13.5.1018 
19. Mazzotti L, Palomo E. A note on the survival of the microfilariae of Mansonella ozzardi. Bull World Health Organ. 1957;16(3):696-699.

20. Drews SJ, Spencer BR, Wendel S, Bloch EM. International Society of Blood Transfusion TTID Working Party Parasite Subgroup. Filariasis and transfusion-associated risk: a literature review. Vox Sang. 2021. doi:10.1111/vox.13073

21. Bregani ER. Filariasis due to blood transfusion. Blood Transfus. 2010;8(2):129. doi:10.2450/2009.0140-09

22. Wiwanitkit V. Filariasis due to blood transfusion: a topic in tropical medicine. Blood Transfus. 2009;7(2):151. doi:10.2450/2008.0041-08

23. Otsuji Y. History, epidemiology and control of filariasis. Trop Med Health. 2011;39(1 Suppl 2):3-13. doi:10.2149/tmh.39-1-suppl_2-3

24. Deribe K, Bakajika DK, Zoure HM-G, Gyapong JO, Molyneux DH, Rebollo MP. African regional progress and status of the programme to eliminate lymphatic filariasis: 2000-2020. Int Health. 2020;13 (Supplement_1):S22-S27. doi:10.1093/inthealth/ihaa058

25. Davies JB. Sixty years of onchocerciasis vector control: a chronological summary with comments on eradication, reinvasion, and insecticide resistance. Annu Rev Entomol. 1994;39:23-45. doi:10.1146/annurev.en.39.010194.000323

26. Lakwo T, Oguttu D, Ukety T, Post R, Bakajika D. Onchocerciasis elimination: progress and challenges. Res Rep Trop Med. 2020;11:81-95. doi:10.2147/RRTM.S224364

27. Crainey JL, Medeiros JF, Pessoa FAC, Luz SLB. Onchocerciasis. Marcondes CB, ed. Springer International Publishing;2017. doi:10.1007/978-3-319-13884-8

28. Bowman DD, Atkins CE. Heartworm biology, treatment, and control. Vet Clin North Am Small Anim Pract. 2009;39(6):1127-1158, vii. doi:10.1016/j.cvsm.2009.06.003

29. Simón F, Siles-Lucas M, Morchón R, et al. Human and animal dirofilariasis: the emergence of a zoonotic mosaic. Clin Microbiol Rev. 2012;25(3):507-544. doi:10.1128/CMR.00012-12

30. American Heartworn Society. American Heartworm Society. American Heartworm Society. Available from: https:/www.heart wormsociety.org/. Accessed March 29, 2021.

31. European Society of Dirofilariosis and Angiostrongylosis. Mission and Objectives of ESDA. ESDA. Available from: https://www.esda. vet/. Accessed March 29, 2021.

32. Companion Animal Parasite Council. Companion Animal Parasite Council. Companion Animal Parasite Council. 2021. Available from: https://capcvet.org/. Accessed March 29.

33. Pulaski CN, Malone JB, Bourguinat C, et al. Establishment of macrocyclic lactone resistant Dirofilaria immitis isolates in experimentally infected laboratory dogs. Parasit Vectors. 2014;7:494. doi:10.1186/ s13071-014-0494-6

34. Bourguinat C, Lee ACY, Lizundia R, et al. Macrocyclic lactone resistance in Dirofilaria immitis: failure of heartworm preventives and investigation of genetic markers for resistance. Vet Parasitol. 2015;210(3-4):167-178. doi:10.1016/j.vetpar.2015.04.002

35. Turner JD, Marriott AE, Hong D, O’ Neill P, Ward SA, Taylor MJ. Novel anti-Wolbachia drugs, a new approach in the treatment and prevention of veterinary filariasis? Vet Parasitol. 2020;279:109057. doi:10.1016/j.vetpar.2020.109057

36. Lane RP, Crosskey RW. Medical Insects and Arachnids. Netherlands: Springer; 1993. doi:10.1007/978-94-011-1554-4

37. Wilson AL, Courtenay O, Kelly-Hope LA, et al. The importance of vector control for the control and elimination of vector-borne diseases. PLoS Negl Trop Dis. 2020;14(1):e0007831. doi:10.1371/ journal.pntd.0007831

38. Simonsen PE, Onapa AW, Asio SM. Mansonella perstans filariasis in Africa. Acta Trop. 2011;120(Suppl 1):S109-120. doi:10.1016/j. actatropica.2010.01.014

39. Shelley AJ, Shelley AJ, Lowry CA, Maia-Herzog M, Dias APAL, Moraes MAP. Biosystematic studies on the Simuliidae (Diptera) of the Amazonia onchocerciasis focus. Bull Natl History Museum Entomol Ser. 1997;66:1-121.
40. Shelley AJ The Blackflies (Diptera: Simuliidae) of Brazil by Shelley, A.j.; Hernandez, L.m.; Maia-herzog, M. Et Al. 2010. Available from: https://www.pemberleybooks.com/product/the-blackflies-dipterasimuliidae-of-brazil/18663/. Accessed February 9, 2021.

41. Yarzábal L, Basáñez MG, Ramírez-Pérez J, Ramírez A, Botto C, Yarzábal A. Experimental and natural infection of Simulium sanchezi by Mansonella ozzardi in the Middle Orinoco region of Venezuela. Trans R Soc Trop Med Hyg. 1985;79(1):29-33. doi:10.1016/00359203(85)90226-3

42. Shelley AJ. Human onchocerciasis in Brazil: an overview. Cad Saude Publica. 2002;18(5):1167-1177. doi:10.1590/s0102311x2002000500009

43. Crosskey RW The natural history of blackflies. 1990. Accessed February 13, 2021. https://www.cabdirect.org/cabdirect/abstract/ 19910502239.

44. Linley JR, Hoch AL, Pinheiro FP. Biting midges (Diptera: Ceratopogonidae) and human health. J Med Entomol. 1983;20 (4):347-364. doi:10.1093/jmedent/20.4.347

45. Lima NF, Veggiani Aybar CA, Dantur Juri MJ, Ferreira MU. Mansonella ozzardi: a neglected New World filarial nematode. Pathog Glob Health. 2016;110(3):97-107. doi:10.1080/ 20477724.2016.1190544

46. Tavares da Silva LB, Crainey JL, Ribeiro da Silva TR, et al. Molecular verification of new world mansonella perstans parasitemias. Emerging Infect Dis. 2017;23(3):545-547. doi:10.3201/eid2303.161159

47. Baia-da-silva DC, Brito-Sousa JD, Rodovalho SR, et al. Current vector control challenges in the fight against malaria in Brazil. Rev Soc Bras Med Trop. 2019;52:e20180542. doi:10.1590/0037-86820542-2018

48. Crainey JL, Costa CHA, de Oliveira Leles LF, et al. Deep-sequencing reveals occult mansonellosis co-infections in residents from the Brazilian Amazon village of São Gabriel da Cachoeira. Clin Infect Dis. 2020. doi:10.1093/cid/ciaa082

49. González MA, Dilger E, Ronderos MM, Spinelli GR, Courtenay O, Hamilton JGC. Significant reduction in abundance of peridomestic mosquitoes (Culicidae) and Culicoides midges (Ceratopogonidae) after chemical intervention in western São Paulo, Brazil. Parasit Vectors. 2020;13(1):549. doi:10.1186/s13071-020-04427-1

50. Brugman VA, England ME, Stoner J, et al. How often do mosquitoes bite humans in southern England? A standardised summer trial at four sites reveals spatial, temporal and site-related variation in biting rates. Parasit Vectors. 2017;10(1):420. doi:10.1186/s13071-0172360-9

51. Harrup L, Miranda M, Carpenter S. Advances in control techniques for Culicoides and future prospects. Vet Ital. 2016;52(3-4):247-264. doi:10.12834/VetIt.741.3602.3

52. Porter JE. Some effects of screens in retarding entry of the common salt-marsh sand fly, Culicoides furens (Poey) (Diptera: heleidae). Mosq News. 1959;19(3):159-163.

53. Picado A, Singh SP, Rijal S, et al. Longlasting insecticidal nets for prevention of Leishmania donovani infection in India and Nepal: paired cluster randomised trial. BMJ. 2010;341:c6760. doi:10.1136/ bmj.c6760

54. Fischer P, Bamuhiiga J, Büttner DW. Treatment of human Mansonella streptocerca infection with ivermectin. Trop Med Int Health. 1997;2(2):191-199. doi:10.1046/j.1365-3156.1997.d01233.x

55. Stensgaard A-S, Vounatsou P, Onapa AW, et al. Ecological drivers of mansonella perstans infection in Uganda and patterns of co-endemicity with lymphatic filariasis and malaria. PLoS Negl Trop Dis. 2016;10(1):e0004319. doi:10.1371/journal.pntd.0004319

56. Molyneux DH, Mitre E, Bockarie MJ, Kelly-Hope LA. Filaria zoogeography in Africa: ecology, competitive exclusion, and public health relevance. Trends Parasitol. 2014;30(4):163-169. doi:10.1016/j.pt.2014.02.002 
57. Plaisier AP, van Oortmarssen GJ, Remme J, Alley ES, Habbema JD. The risk and dynamics of onchocerciasis recrudescence after cessation of vector control. Bull World Health Organ. 1991;69 (2):169-178

58. Hamley JID, Milton P, Walker M, Basáñez M-G. Modelling exposure heterogeneity and density dependence in onchocerciasis using a novel individual-based transmission model, EPIONCHO-IBM: implications for elimination and data needs. PLoS Negl Trop Dis. 2019;13(12): e0007557. doi:10.1371/journal.pntd.0007557

59. Boatin BA, Richards FO. Control of onchocerciasis. Adv Parasitol. 2006;61:349-394. doi:10.1016/S0065-308X(05)61009-3

60. Norman RA, Chan MS, Srividya A, et al. EPIFIL: the development of an age-structured model for describing the transmission dynamics and control of lymphatic filariasis. Epidemiol Infect. 2000;124 (3):529-541. doi:10.1017/s0950268899003702

61. Koala L, Nikiema A, Post RJ, et al. Recrudescence of onchocerciasis in the Comoé valley in Southwest Burkina Faso. Acta Trop. 2017;166:96-105. doi:10.1016/j.actatropica.2016.11.003

62. Sheel M, Sheridan S, Gass K, et al. Identifying residual transmission of lymphatic filariasis after mass drug administration: comparing school-based versus community-based surveillance - American Samoa, 2016. PLoS Negl Trop Dis. 2018;12(7):e0006583. doi:10.1371/journal.pntd.0006583

63. Ta T-H, Moya L, Nguema J, et al. Geographical distribution and species identification of human filariasis and onchocerciasis in Bioko Island, Equatorial Guinea. Acta Trop. 2018;180:12-17. doi:10.1016/j.actatropica.2017.12.030

64. Molyneux DH. Advancing toward the Elimination of Lymphatic Filariasis. $N$ Engl J Med. 2018;379(19):1871-1872. doi:10.1056/ NEJMe1811455

65. Sauerbrey M, Rakers LJ, Richards FO. Progress toward elimination of onchocerciasis in the Americas. Int Health. 2018;10(suppl_1):i71i78. doi:10.1093/inthealth/ihx039

66. Wanji S, Nji TM, Hamill L, et al. Implementation of test-and-treat with doxycycline and temephos ground larviciding as alternative strategies for accelerating onchocerciasis elimination in an area of loiasis co-endemicity: the COUNTDOWN consortium multi-disciplinary study protocol. Parasit Vectors. 2019;12(1):574. doi:10.1186/s13071-019-3826-8

67. Jourdan PM, Lamberton PHL, Fenwick A, Addiss DG. Soiltransmitted helminth infections. Lancet. 2018;391(10117):252-265. doi:10.1016/S0140-6736(17)31930-X

68. Hotez PJ, Bundy DAP, Beegle K, et al.Helminth Infections: soiltransmitted Helminth Infections and Schistosomiasis. Jamison DT, Breman JG, Measham AR, et al., editors. Disease Control Priorities in Developing Countries. 2nd ed. World Bank;2006. Accessed February 10, 2021. http://www.ncbi.nlm.nih.gov/books/NBK11748/.

69. Walker M, Stolk WA, Dixon MA, et al. Modelling the elimination of river blindness using long-term epidemiological and programmatic data from Mali and Senegal. Epidemics. 2017;18:4-15. doi:10.1016/j. epidem.2017.02.005

70. The Ivermectin Roadmappers, Billingsley P, Binka Fet al. A roadmap for the development of ivermectin as a complementary malaria vector control tool. Am J Trop Med Hyg. 2020;102(2s):3-24. doi:10.4269/ ajtmh.19-0620.

71. Pinilla YT, Lopes CP, Sampaio SV, et al. Promising approach to reducing Malaria transmission by ivermectin: sporontocidal effect against Plasmodium vivax in the South American vectors Anopheles aquasalis and Anopheles darlingi. PLoS Negl Trop Dis. 2018;12(2):e0006221. doi:10.1371/journal.pntd.0006221

72. de Basano SA, Fontes G, Medeiros JF, et al. Sustained clearance of Mansonella ozzardi infection after treatment with ivermectin in the Brazilian Amazon. Am J Trop Med Hyg. 2014;90(6):1170-1175. doi:10.4269/ajtmh.13-0410
73. Perez-Garcia LA, Mejias-Carpio IE, Delgado-Noguera LA, et al. Ivermectin: repurposing a multipurpose drug for Venezuela's humanitarian crisis. Int $J$ Antimicrob Agents. 2020;56(2):106037. doi:10.1016/j.ijantimicag.2020.106037

74. Metzger WG, Theurer A, Pfleiderer A, et al. Ivermectin for causal malaria prophylaxis: a randomised controlled human infection trial. Trop Med Int Health. 2020;25(3):380-386. doi:10.1111/tmi.13357

75. Bakowski MA, McNamara CW. Advances in antiwolbachial drug discovery for treatment of parasitic filarial worm infections. Trop Med Infect Dis. 2019;4:3. doi:10.3390/tropicalmed4030108

76. Taylor MJ, von Geldern TW, Ford L, et al. Preclinical development of an oral anti-Wolbachia macrolide drug for the treatment of lymphatic filariasis and onchocerciasis. Sci Transl Med. 2019;11:483. doi:10.1126/scitranslmed.aau2086

77. von Geldern TW, Morton HE, Clark RF, et al. Discovery of ABBV-4083, a novel analog of Tylosin A that has potent anti-Wolbachia and anti-filarial activity. PLoS Negl Trop Dis. 2019;13(2):e0007159. doi:10.1371/journal.pntd.0007159

78. Taylor MJ, Hoerauf A, Townson S, Slatko BE, Ward SA. AntiWolbachia drug discovery and development: safe macrofilaricides for onchocerciasis and lymphatic filariasis. Parasitology. 2014;141 (1):119-127. doi:10.1017/S0031182013001108

79. Taylor EM. NTD Diagnostics for Disease Elimination: a Review. Diagnostics (Basel). 2020;10:6. doi:10.3390/diagnostics10060375

80. Tylamac (ABBV-4083) DNDi. 2015. Available from: https://dndi. org/research-development/portfolio/abbv-4083/. Accessed March 30, 2021.

81. Bakowski MA, Shiroodi RK, Liu R, et al. Discovery of short-course antiwolbachial quinazolines for elimination of filarial worm infections. Sci Transl Med. 2019;11:491. doi:10.1126/scitranslmed. aav3523

82. Hübner MP, Gunderson E, Vogel I, et al. Short-course quinazoline drug treatments are effective in the Litomosoides sigmodontis and Brugia pahangi jird models. Int J Parasitol Drugs Drug Resist. 2020;12:18-27. doi:10.1016/j.ijpddr.2019.12.001

83. Grobusch MP, Kombila M, Autenrieth I, Mehlhorn H, Kremsner PG. No evidence of Wolbachia endosymbiosis with Loa loa and Mansonella perstans. Parasitol Res. 2003;90(5):405-408. doi:10.1007/s00436-003-0872-z

84. Rasgon JL, Scott TW. Phylogenetic characterization of Wolbachia symbionts infecting Cimex lectularius L. and Oeciacus vicarius Horvath (Hemiptera: cimicidae). J Med Entomol. 2004;41 (6):1175-1178. doi:10.1603/0022-2585-41.6.1175

85. Lefoulon E, Bain O, Makepeace BL, et al. Breakdown of coevolution between symbiotic bacteria Wolbachia and their filarial hosts. PeerJ. 2016;4:e1840. doi:10.7717/peerj.1840

86. Batsa Debrah L, Phillips RO, Pfarr K, et al. The efficacy of doxycycline treatment on mansonella perstans infection: an open-label, randomized trial in Ghana. Am J Trop Med Hyg. 2019;101 (1):84-92. doi:10.4269/ajtmh.18-0491

87. Coulibaly YI, Dembele B, Diallo AA, et al. A randomized trial of doxycycline for Mansonella perstans infection. $N \mathrm{Engl} \mathrm{J} \mathrm{Med.}$ 2009;361(15):1448-1458. doi:10.1056/NEJMoa0900863

88. Gehringer C, Kreidenweiss A, Flamen A, Antony JS, Grobusch MP, Bélard S. Molecular evidence of Wolbachia endosymbiosis in Mansonella perstans in Gabon, Central Africa. $J$ Infect Dis. 2014;210(10):1633-1638. doi:10.1093/infdis/jiu320

89. Leles LF. Avaliação da Diversidade Genética Populacional e Prevalência de Infecção por Wolbachia em Mansonella ozzardi no Estado do Amazonas, Brasil. 2019. Available from: https://www.arca. fiocruz.br/handle/icict/32209. Accessed March 30, 2021.

90. Ferri E, Bain O, Barbuto M, et al. New insights into the evolution of Wolbachia infections in filarial nematodes inferred from a large range of screened species. PLoS One. 2011;6(6):e20843. doi:10.1371/journal.pone. 0020843 
91. Correa CC, Ballard JWO. Wolbachia associations with insects: winning or losing against a master manipulator. Front Ecol Evol. 2016;3. doi:10.3389/fevo.2015.00153

92. Nikoh N, Hosokawa T, Moriyama M, Oshima K, Hattori M, Fukatsu T. Evolutionary origin of insect-Wolbachia nutritional mutualism. Proc Natl Acad Sci U S A. 2014;111(28):10257-10262. doi:10.1073/pnas.1409284111

93. Medeiros JF, Almeida TAP, Silva LBT, et al. A field trial of a PCRbased Mansonella ozzardi diagnosis assay detects high-levels of submicroscopic M. ozzardi infections in both venous blood samples and FTA $^{\circledR}$ card dried blood spots. Parasit Vectors. 2015;8(1):280. doi:10.1186/s13071-015-0889-z

94. Romão Ribeiro da Silva T, Crainey JL, Costa pessoa FA, et al. Blackflies in the ointment: o. volvulus vector biting can be significantly reduced by the skin-application of mineral oil during human landing catches. PLoS Negl Trop Dis. 2019;13(4):e0007234. doi:10.1371/journal.pntd.0007234
95. Post RJ, Crainey JL, Bivand A, Renz A. Laser-assisted microdissection for the study of the ecology of parasites in their hosts. Mol Ecol Resour. 2009;9(2):480-486. doi:10.1111/j.1755-0998.2008.02437.x

96. Osborne LC, Monticelli LA, Nice TJ, et al. Coinfection. Virus-helminth coinfection reveals a microbiota-independent mechanism of immunomodulation. Science. 2014;345 (6196):578-582. doi:10.1126/science.1256942

97. Hartmann W, Brunn M-L, Stetter N, et al. Helminth infections suppress the efficacy of vaccination against seasonal influenza. Cell Rep. 2019;29(8):2243-2256.e4. doi:10.1016/j.celrep.2019.10.051

\section{Publish your work in this journal}

Research and Reports in Tropical Medicine is an international, peerreviewed, open access journal publishing original research, case reports, editorials, reviews and commentaries on all areas of tropical medicine, including: Diseases and medicine in tropical regions; Entomology; Epidemiology; Health economics issues; Infectious disease; Laboratory science and new technology in tropical medicine;
Parasitology; Public health medicine/health care policy in tropical regions; and Microbiology. The manuscript management system is completely online and includes a very quick and fair peer-review system. Visit http://www.dovepress.com/testimonials.php to read real quotes from published authors. 